\section{Improving the interaction between the ophthalmology and histopathology departments}

A Maudgil', SM Salvi ${ }^{1}$, JHY Tan ${ }^{1}$ and HS Mudhar ${ }^{2}$

Keywords: ophthalmic histopathology;

communication; histopathology request form

\section{Introduction}

The ophthalmic histopathology service at the Royal Hallamshire Hospital, Sheffield receives over 1500 specimens a year of which approximately 700 are generated from the ophthalmology department. In order to maximise the efficiency and effectiveness of this service, good communication between the ophthalmologist and the histopathologist via the histopathology request form is key. If this form is not filled out appropriately, errors and inefficiencies can result, including insufficient patient details necessary to log a case for histopathological reporting or insufficient doctor details preventing the histopathologist from being able to contact the relevant team about results. This in turn results in wastage of valuable clinical time by histopathologists and their secretaries chasing such necessary information.

The main aim of this large audit was to improve this communication between the two departments by optimising the ophthalmic histopathology request form, making it easier to fill out by the operating ophthalmologist and hence more consistently completed correctly. We also aimed to increase awareness among ophthalmology staff of the importance of filling out the histopathology request form as fully and correctly as possible, to provide the most comprehensive information for the histopathologist and minimise the chance of medical error.

\section{Materials and methods}

Before commencing this work, the audit was registered with the Clinical Effectiveness Department, Royal Hallamshire Hospital.
Received: 23 February 2011 Published online: 13 May 2011
Eye (2011) 25, 998-1004; doi:10.1038/eye.2011.96;

published online 13 May 2011 
A proforma for data collection was created in Microsoft Access based on the boxes to be completed in original histopathology request form, Form 1 (see Figures 1 and 2). This was used to document whether each section of the form had been completed fully. In each section the answer selection was either 'yes' (indicating that box had been completed) or 'no' (indicating that box had been left blank). The exceptions to this were the sections for ophthalmic, general medical, and drug history, in which information was classified as 'adequate', 'inadequate', or 'absent'. A judgement was made over whether information was adequate by a single medical reviewer (AM). For the sections 'specimen site' and 'specimen type', text boxes were created in the proforma for this to be completed.

All the forms received by the department of histopathology from the department of ophthalmology between June 2005 and July 2006 inclusive were reviewed; a total of 710 . Histopathology request forms for specimens received from peripheral units were excluded.

Once collected, data were analysed and summarised for departmental presentation. The results were used to identify sections of the form that were consistently not being filled out correctly. This information, together with the comments from the departmental presentation and input from the histopathology department, was used to re-model a draft of a new ophthalmic histopathology request form, Form 2 with a more systematic, userfriendly format. The draft of Form 2 was then circulated by email around users in the department for further comments and suggestions. Specific suggestions and concerns elicited from this were addressed and a final form was created (Figure 3). This was circulated into the department for general use.

One year after introduction of the new ophthalmic histopathology request form, the audit loop was closed, by auditing completion of the new improved form. Consecutive forms from a 3-month period received by the histopathology department from the ophthalmology department from January, February and March 2008 were reviewed. Results were analysed and compared with previous data.

\section{Results}

A total of 710 histopathology request forms were audited for the period between July 2005 and June 2006 inclusive. Results of compliance with completion of each section of the original form are given in Table 1. The front of the new form, Form 2 that was designed is shown in Figure 3. The diagrams on the back of the original form, shown in Figure 2 were not changed. Compliance with each section of the new form is also given in Table 1. Comparison of data between the two forms shows that each section of the new form was completed with a higher percentage or, in the cases of the boxes 'signature' and 'drug history', at least similar percentage after re-design of the form.

\section{Discussion}

The aim of this audit was to improve the ophthalmic histopathology request form and its completion by ophthalmology staff at the Royal Hallamshire Hospital, Sheffield. Improving this written communication is important for a number of reasons. Firstly, maximising the relevant clinical information available to the histopathologist when specimens are reported enables them to investigate the specimen appropriately with various stains, and provide the most likely clinical diagnosis and possible differentials, which allow clinicians to plan appropriate patient management. Equally, non-clinical sections of the form, such as patient details, are important to fill in to create an accurate histological and clinical record of the patient for safety and medicolegal reasons, and to ensure the correct decisions are made about the correct patients. Similarly, the provision of correct contact information for the operating doctor is vital for the histopathologist to be able to relay pertinent information that will affect urgent clinical decisions such as results of temporal artery biopsy (TAB) or clearance margins for staged tumour excision.

The results of this study show that we were successful in improving completion of every section of the form by the audit process. Our main approach to this aim was to improve the ophthalmic histopathology form. We considered the most valuable feedback on improving the form would be gained by asking the users themselves. This was achieved by a cycle of gathering opinions and suggestions for improvement, initially in an open forum at a departmental meeting where the audit results were presented. This meeting also served to educate and raise awareness on the importance of filling out the form correctly and shortcomings that had occurred.

Shortcomings in the completion of the initial histopathology request form were identified in the results in every section of the form. A high percentage of forms $(93.2 \%)$ had a patient sticker in the results from Form 1 but there were four forms that lacked the minimum data set of patient name, hospital number and date of birth. Such specimens cannot be processed by the histopathology department, which has ethical as well as clinical implications. None of the new forms were submitted without the minimum data set, which may have been achieved by increasing awareness on the importance of filling out these forms, or could be due to the smaller sample size of the new forms. Similarly, the proportion of patients with adequate ophthalmic history was improved in the new forms, achieved largely by 
The Directorate is committed to providing a service of the highest quality and is registered with The National Accreditation Body. For the current accreditation status of the individual departments please see: www.cpa-uk.co.uk

\section{Sheffield Teaching Hospitals W/HE \\ 灾 \\ LABORATORY MEDICINE DIRECTORATE OPHTHALMIC PATHOLOGY REQUEST FORM A LABORATORY OF THE NATIONAL SPECIALIST OPHTHALMIC PATHOLOGY SERVICE (PLEASE SEE REVERSE FOR LABORATORY DETAILS)}

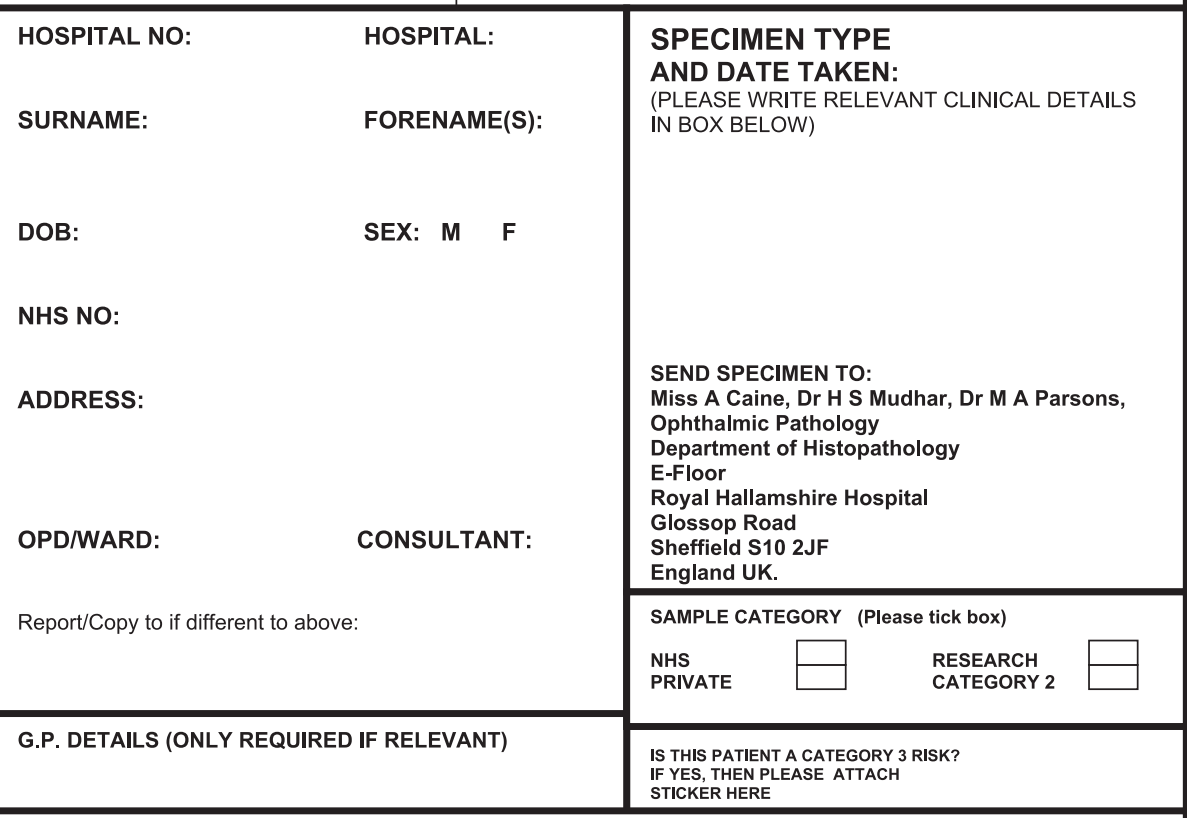

RELEVANT OPHTHALMIC, GENERAL MEDICAL AND DRUG HISTORY Please write clearly Use ophthalmic diagrams overleaf if required

Signature \& Date:

Requesting Clinician (BLOCK CAPITALS) and Bleep No.

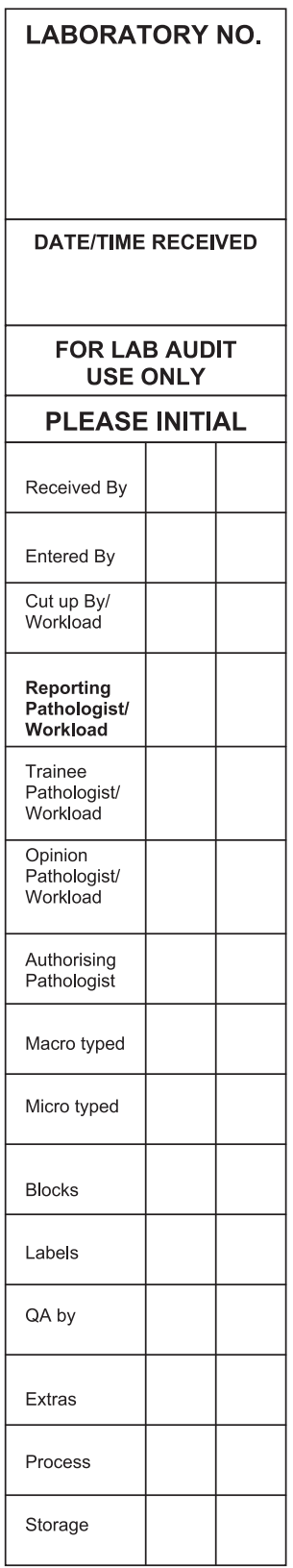

LABORATORY NOTES

Figure 1 Form 1: Front of original histopathology request form. 
RIGHT

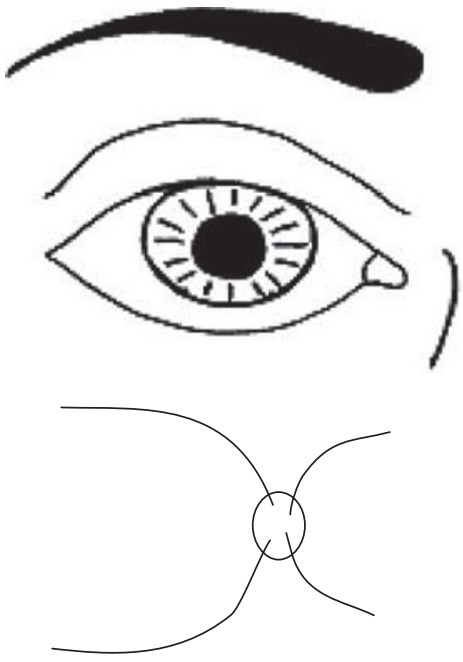

ANTERIOR VIEW

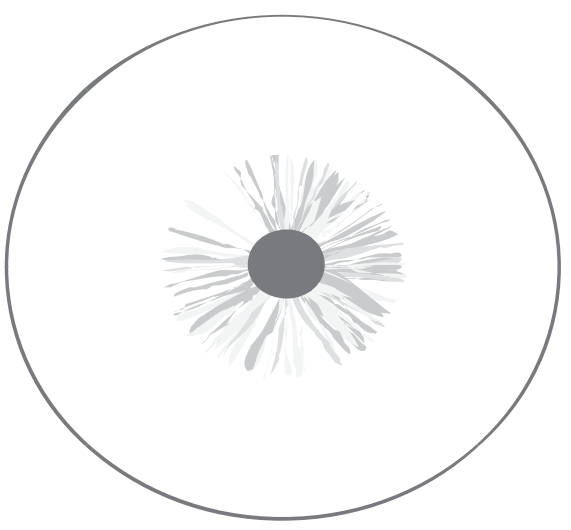

LEFT
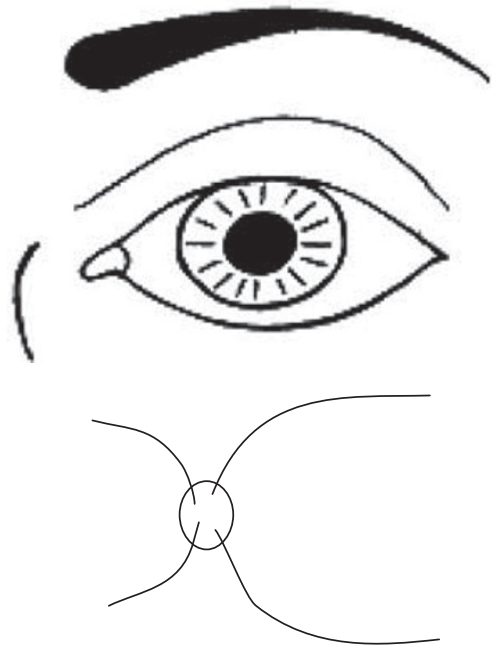

VIEW FROM ABOVE

$\mathbf{L}$

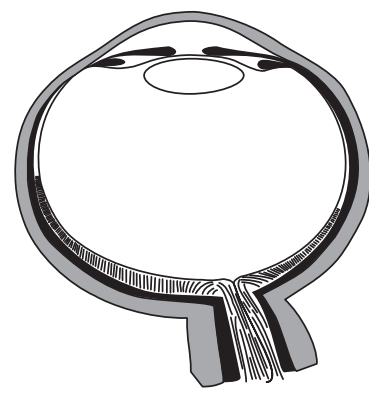

$\mathbf{R}$

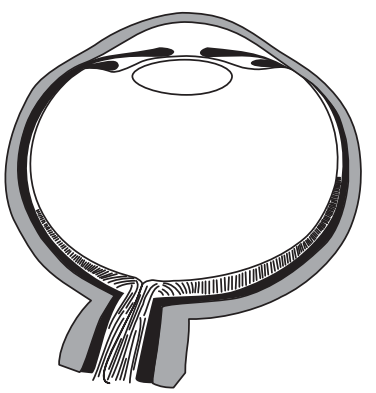

Laboratory Medicine Directorate GENERAL ENQUIRIES AND RESULTS

\begin{tabular}{|lr|}
\hline \multicolumn{2}{|c|}{$\begin{array}{c}\text { Northern General Hospital } \\
\text { Herries Road, Sheffield S5 7AU }\end{array}$} \\
Clinical Chemistry & 2714716 \\
Coagulation & 2714943 \\
Haematology & 2714304 \\
Histopathology & $\mathbf{2 7 1 6 3 2 1}$ \\
Immunology & 2715552 \\
Microbiology & 2714777 \\
HOSPITAL SWITCHBOARD & 2434343 \\
\hline
\end{tabular}

\begin{tabular}{|lc|}
\hline \multicolumn{2}{|c|}{$\begin{array}{c}\text { Royal Hallamshire Hospital } \\
\text { Glossop Road, Sheffield S10 2JF }\end{array}$} \\
Clinical Chemistry & 2712348 \\
Coagulation & 2712955 \\
Haematology & 2712284 \\
Histopathology & $\mathbf{2 7 1 2 7 2 8}$ \\
Microbiology & 2713126 \\
HOSPITAL SWITCHBOARD & 2711900 \\
\hline
\end{tabular}

Phlebotomy/GP Services - OPD

Monday-Friday $9.00 \mathrm{am}$ to $5.00 \mathrm{pm}$

C Floor OPD Phlebotomy/GP Services 2713072 Monday - Friday $8.30 \mathrm{am}$ to $5.30 \mathrm{pm}$

SEE LABORATORY HANDBOOK FOR DETAILS OF LABORARY SERVICES

OR http://195.106.36.15/LabMed nhs/Pages/intro.htm

SPECIMEN LABEL - Please label all specimens clearly, giving the patient's name, date of birth, requesting doctor and destination. UNLABELLED SPECIMENS WILL NOT BE PROCESSED

Multiple specimens may be sent with one request form.

HIGH RISK SPECIMENS - Specimens from patients with known or suspected infection with TB, Hepatitis, HIV, etc. are especially hazardous and must be identified as such with YELLOW CATEGORY 3 LABELS on specimen containers and request forms.

Figure 2 Form 1: Back of original histopathology request form. 


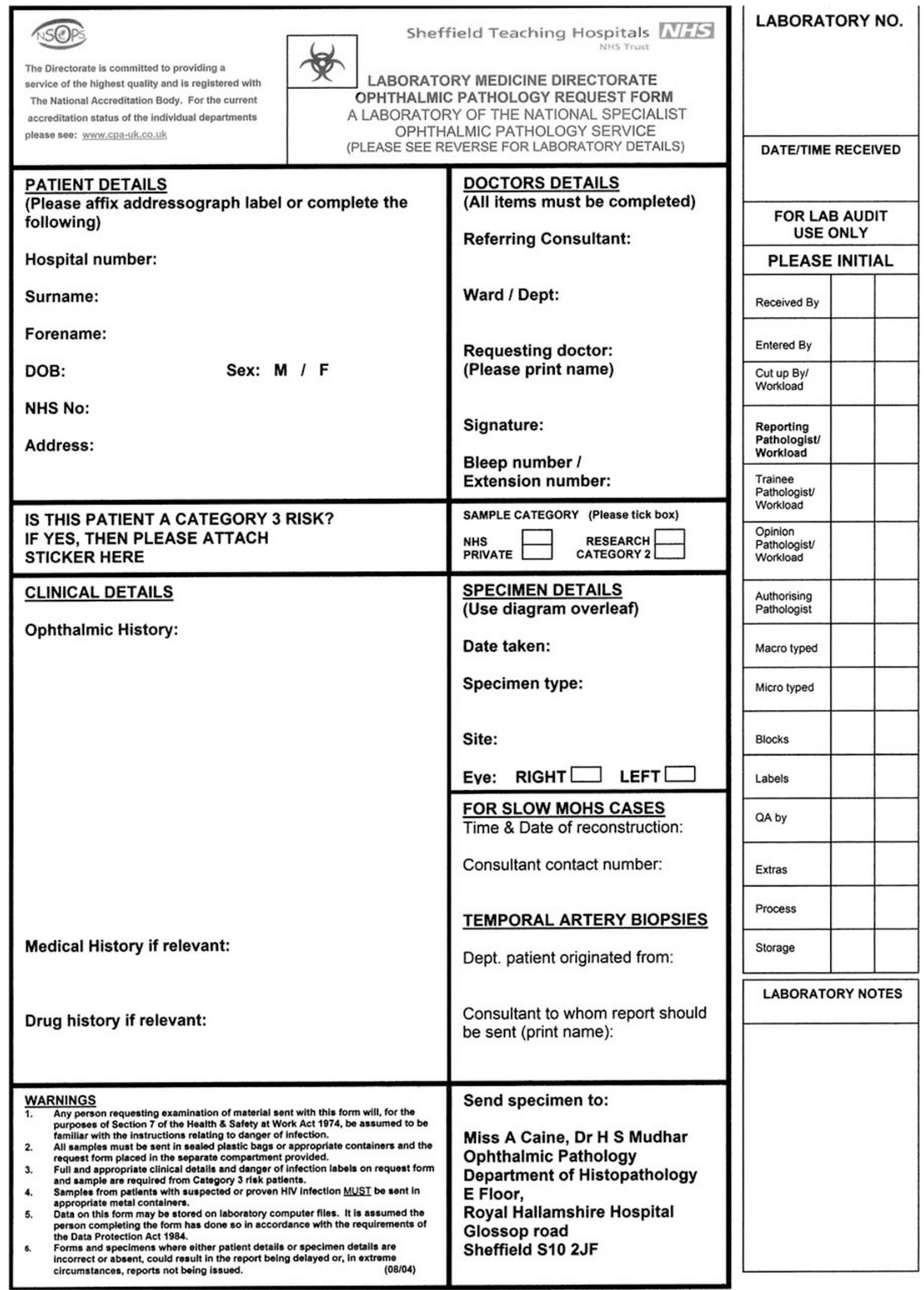

Figure 3 Form 2: Front of new histopathology request form. 
Table 1 Results comparing percentage completion of Form 1 vs Form 2

\begin{tabular}{lcc}
\hline Section of form & $\begin{array}{c}\text { Form 1 } \\
\text { completion } \\
(\mathrm{n}=710)\end{array}$ & $\begin{array}{r}\text { Form } 2 \\
\text { completion } \\
(\mathrm{n}=224)\end{array}$ \\
\hline Patient sticker & $93.2 \%$ & $95.9 \%$ \\
If no sticker, forms without & 4 of 48 & 0 of 9 \\
minimum data set? & & \\
Ward/OPD & $50.6 \%$ & $96.4 \%$ \\
Referring consultant & $80.1 \%$ & $99.6 \%$ \\
Operating doctor & $85.6 \%$ & $95.9 \%$ \\
Signature & $97.2 \%$ & $97.8 \%$ \\
Contact number & $50.3 \%$ & $82.5 \%$ \\
Sufficient ophthalmic history & $84.1 \%$ & $98.2 \%$ \\
Medical history & $9.8 \%$ & $14.2 \%$ \\
Drug history & $3.9 \%$ & $4.0 \%$ \\
Date taken & $64.4 \%$ & $93.0 \%$ \\
Side & $88.2 \%$ & $98.0 \%$ \\
Diagrams marked & $48.2 \%$ & $71.4 \%$ \\
\hline
\end{tabular}

education in the department, as the format of this part of the form remained relatively unchanged.

Completion of many sections of the form was increased by the new format of the form, making it more user-friendly and coherent. Mainly this was achieved by grouping boxes together under relevant headings; 'Patient details', 'Doctors details' and 'Specimen details'. Using this approach, the filling out of essential information such as contact details of the operating doctor was improved to $82.5 \%$, having been missed in $50 \%$ of the initial forms. More modest, but evident improvements in filling out of 'Referring consultant' (80.1-99.6\%) and 'Operating doctor' (85.6-95.9\%) were also seen. Less important information for the histopathologist in this section such as 'Ward/OPD' also saw dramatic improvements with the re-formatting, its percentage completion almost doubled.

Further important data items frequently being missed on the original form were 'Date taken' and 'Side', that is, laterality. Re-organising these, each with its own space under 'Specimen details', allowed the completion of 'Date taken' to be significantly improved from 60.4 to $93.0 \%$. Including a tick box for laterality in this section also proved very useful in improving completion of this to $98.0 \%$, which on the initial form was perhaps unexpectedly low (at $88.2 \%$ ), given that all ophthalmic specimens should specify laterality for safety reasons. The exceptions to this would be midline facial lesions, on the forehead or bridge of nose.

As expected, there was not much percentage increase in the medical and drug history that was given between the two forms as these are only requested if relevant, and in many cases they are not. Specific examples where they may be relevant include syndromes associated with ophthalmic lesions and whether steroids have already been started in specimens sent for diagnosis of temporal arteritis.
The structure of the diagrams on the back of the form were not changed, but the proportion of forms with these completed was significantly improved from 48.2 to $71.4 \%$, which again can be accounted for by increased awareness through education of users.

At the histopathologist's request two new boxes were included in the new form for TAB and multistage eyelid tumour excision cases respectively. For all such cases (6 TABs and 15 multistage eyelid tumour excision cases), these sections were completed appropriately in the 224 samples of new forms.

To our knowledge, this is the first such study that has been performed specifically in ophthalmic histopathology. Similar work on the generic pathology request form has been published, which also found that doctor contact details were frequently being missed, ${ }^{1}$ as well as doctor name and signature. ${ }^{2}$ This second study by Burnett et al found that by introducing self-inking stamps the proportion of doctor details missed could be reduced from 43 to $2 \%$, but we have achieved comparable outcomes with our strategy of improving the form and user awareness. A further study by Zemlin $e t a l^{3}$ in the field of endocrinology confirms that neglecting to give relevant drug information on the laboratory request form limits the pathologists ability to infer the correct clinical diagnosis. Likewise, in ophthalmology failing to give such information on the use of steroids in $\mathrm{TAB}$, or previous pharmacological and topical therapies on other lesions hampers the histopathologist in a similar manner.

In conclusion, modifications of the histopathology request form through the audit process, together with increasing awareness amongst users in the ophthalmology department, have enabled us to fulfil our aim of improving filling out of histopathology request forms to strive for better communication between the two departments-a small but significant step that is certain to help us fulfil our ultimate goal, improved safety and efficiency in the delivery of care for the ophthalmic patient.

\section{Summary}

\section{What was known before}

- Poor communication between departments of ophthalmology and histopathology can compromise patient safety and clinical efficiency in turnaround times.

- Certain important sections of the histopathology request form are frequently not filled in appropriately.

\section{What this study adds}

- Comprehensive review of the shortcomings of the written communication between the departments of ophthalmology and histopathology through the process of audit.

- Evidence of improved communication to the satisfaction of histopathologist.

- A template for the ophthalmic histopathology request form. 


\section{Conflict of interest}

The authors declare no conflict of interest.

\section{References}

1 Nutt L, Zemlin AE, Erasmus RT. Incomplete laboratory request forms: the extent and impact on critical results

at a tertiary hospital in South Africa. Ann Clin Biochem 2008; 45: 463-466.

2 Burnett L, Chesher D, Mudaliar Y. Improving the quality of information on pathology request forms. Ann Clin Biochem 2004; 41: 53-56.

3 Zemlin AE, Nutt L, Burgess LJ, Eiman F, Erasmus RT. Potential for medical error: incorrectly completed request forms for thyroid function tests limit pathologists' advice to clinicians. S Afr Med J 2009; 99(9): 668-671. 